\title{
L'Homme
}

L HDME Revue française d'anthropologie

\section{Les techniques paradoxales ou l'inefficacité technique voulue}

Paradoxical Techniques, or the Requisite Technical Ineffectiveness

\section{Catherine Tourre-Malen}

\section{(2) OpenEdition}

Journals

\section{Édition électronique}

URL : http://journals.openedition.org/lhomme/22906

DOI : 10.4000/lhomme.22906

ISSN : 1953-8103

Éditeur

Éditions de l'EHESS

\section{Édition imprimée}

Date de publication : 21 novembre 2011

Pagination : 203-226

ISSN : 0439-4216

\section{Référence électronique}

Catherine Tourre-Malen, « Les techniques paradoxales ou l'inefficacité technique voulue », L'Homme

[En ligne], 200 | 2011, mis en ligne le 09 novembre 2013, consulté le 03 mai 2019. URL : http:// journals.openedition.org/lhomme/22906 ; DOI : 10.4000/lhomme.22906

Ce document a été généré automatiquement le 3 mai 2019.

(c) École des hautes études en sciences sociales 


\title{
Les techniques paradoxales ou l'inefficacité technique voulue
}

\author{
Paradoxical Techniques, or the Requisite Technical Ineffectiveness
}

Catherine Tourre-Malen

1 MARCel mauss définissait les techniques comme «actes traditionnels efficaces » (Mauss 1966 [1936]). Mais toutes les techniques sont-elles toujours efficaces? Sinon, à quelles logiques répondent-elles? Ces questions ont commencé à m'apparaître lorsque, étudiant l'évolution des techniques féminines d'équitation (Tourre-Malen 2006b), j'ai été amenée à m'intéresser à la monte en amazone, c'est-à-dire à cette façon d'aller à cheval, les deux jambes du même côté, dont le manque de commodité était reconnu et qui a néanmoins perduré durant plusieurs siècles, malgré la coexistence d'une technique plus efficace : la monte à califourchon. Je me trouvais là devant une «donnée inattendue, aberrante et capitale» (Merton 1997 [1957] : 43) : alors que, selon André Leroi-Gourhan dans son analyse des phénomènes de diffusion, «on n'échange pas la charrue contre la houe » (Leroi-Gourhan 1945 : 304), il semblait bien que, dans le cas des techniques équestres, au sein de la même société, on ait conservé et amélioré la « houe » quoique la « charrue » fût à disposition, mais réservée aux hommes. Cette donnée prit un tour nouveau quand je commençai à m'intéresser à la marche avec des talons hauts (Tourre-Malen 2009 et 2012a) et que j'y relevai des traits communs avec la monte en amazone. Certes, deux techniques singulières ne font pas le printemps, mais elles piquèrent assez ma curiosité pour me lancer ${ }^{1}$ sur la piste des techniques qui constituent une entorse à la "suite d'états de mieux en mieux adaptés qui ", selon André Leroi-Gourhan, "illustrent le progrès " (1945 : 304). Dans un premier temps, je mettrai en évidence l'inefficacité pratique de la monte en amazone et de la marche en talons, et retracerai, par là, l'amorce de ma réflexion. Dans un deuxième temps, je m'efforcerai d'élargir l'échantillon et d'établir une typologie de ces techniques qui limitent l'emprise humaine sur la nature. Enfin, je tenterai de trouver un sens à l'ensemble: quelles fonctions remplit le paradoxe qui caractérise ces techniques ? À quelles logiques répond-il ? 


\section{De la monte en amazone à la marche en talons hauts : des techniques pas comme les autres}

\section{La monte en amazone}

2 Compte tenu de la place du cheval dans la société occidentale avant l'ère industrielle, les femmes, ou au moins celles appartenant aux élites, ont, depuis toujours, eu accès à l'équitation, et cela même dans les "sociétés à écuyers " ${ }^{2}$. Jusqu'à la fin du Moyen Âge, pour aller à cheval, les femmes purent recourir à deux techniques différentes de monte : soit à califourchon, soit les deux jambes tombant du même côté de la monture. Cette dernière technique s'imposa alors, au détriment de la première (Closson 1992 : 62-67). En effet, à partir du XVI ${ }^{\mathrm{e}}$ siècle, chevaucher les cuisses ouvertes ne fut plus considéré comme convenable pour une femme (Nelson 1994: 103), tendance qui s'accentua au cours des siècles sous la pression des normes sociales: en 1914, il paraissait encore inconvenant qu'une femme montât autrement (Bouchet $1993: 258$ ).

3 Au fil du temps, cette " équitation des dames $»^{3}$ ou «monte en amazone " connut de nombreux perfectionnements dont les plus déter-minants furent l'invention de la selle dite "à corne" ( $\mathrm{xv}^{\mathrm{e}}$ siècle), puis de la selle à deux fourches ( $\mathrm{xvI}^{\mathrm{e}}$ siècle) et, enfin, la découverte de la troisième fourche (1830)(Tourre-Malen 2006b : 22-25). Ces améliorations firent passer les femmes d'une position qui leur permettait à peine d'encaisser les allures modérées d'une monture confortable, à une position qui leur procurait une solidité suffisante pour résister aux allures vives (Ibid.), dont témoigne la virtuosité des écuyères, figures emblématiques de l'équitation de spectacle du XIX siècle (Nelson $1994: 103$ ).

Mais, même à son acmé, cette façon de monter à cheval se révélait bien moins efficace que la monte à califourchon pour contrôler et obtenir des performances d'une monture. En effet, avoir les deux jambes du même côté, même maintenues dans les fourches, donne une assise moins stable que lorsqu'on enserre le cheval entre ses jambes. La chute était d'ailleurs considérée, à juste titre, plus périlleuse pour les amazones que pour les hommes (Anonyme 1918 : 204), ce que de tragiques accidents confirment (Bouchet $1993: 283$ ). Les techniciens équestres d'alors étaient conscients du moindre contrôle qu'offrait la monte en amazone et de sa dangerosité potentielle ; aussi conseillaient-ils de n'utiliser que des montures «sages» (Vergnaud 1842: 187; François Baucher [1851] cité in Cabaud 1986 : 87). Les écuyères de l'époque reconnaissaient qu'elles se trouvaient dans la «presque impossibilité de faire obéir un cheval, à moins qu'il ne soit complètement rompu à ce qu'on lui demande" (Anonyme 1918: 210). Tombée en désuétude à partir des années 1930, la pratique de la monte en amazone perdure à l'heure actuelle de façon confidentielle (Tourre-Malen 2006b : chap. I). Sa dimension limitante est prise en compte par la Fédération française d'équitation, puisque le règlement n'autorise que certaines compétitions en amazone et impose la monte à califourchon pour les épreuves de cross ${ }^{4}$ des brevets fédéraux d'amazone (Fédération française d'équitation $2008: 1)^{5}$.

Force est de constater l'étrangeté de cet acharnement à perfectionner, durant des siècles, une technique incommode pour la maîtrise de l'animal et qui s'avérait même dangereuse, alors que l'on disposait d'une technique alternative à l'efficacité éprouvée. 


\section{La marche en talons hauts}

5 À l'instar de la monte en amazone, la marche en talons hauts représente un curieux choix technique. De même que la première limitait le contrôle d'un cheval, la seconde limite la marche, alors qu'une autre technique, utilisant d'autres instruments, à l'efficacité reconnue, coexiste.

Marche à plat et marche en talons cohabitent en Europe depuis le XVII siècle, époque où apparurent les « souliers à pont-levis » ou les « souliers à cric »", c'est-à-dire des souliers qui présentaient un vide entre le talon et la partie antérieure de la semelle (Laforge 2001 : 53). Massifs et élevés, les talons ornaient les bottes masculines et restaient assez lourds dans la chaussure féminine. C'est à partir du XVIII ${ }^{\mathrm{e}}$ siècle, qu'allégés et incurvés (Bossan 2004 : 51), ils firent partie du "véritable charme d'une femme $»^{7}$, tandis que les hommes, eux, portaient des chaussures à talons plats. Les talons hauts restaient toutefois réservés aux couches les plus élevées de la société, les classes populaires se contentant le plus souvent de grossiers sabots ou, au mieux, de modestes souliers qui étaient portés jusqu'à l'usure totale (Ibid.: 65). Les années 1950 furent marquées par l'invention des talons aiguilles, les talons les plus fins connus jusqu'alors (Ibid. : 76). Ils rencontrèrent un succès sans précédent (Laforge 2001 : 57). L'époque actuelle voit une grande variété de formes et de hauteurs de talons. On perçoit toutefois un certain engouement pour les talons hauts (plus de sept centimètres) ${ }^{8}$, même si, dans la pratique quotidienne, le nombre de "femmes à chaussures plates» l'emporte sur celui des "femmes à talons hauts». Ces catégories se révèlent d'ailleurs en grande partie poreuses: sauf à être une inconditionnelle des chaussures plates ou, au contraire, des talons hauts, toute femme peut changer de registre en fonction des circonstances et/ou de ses envies du moment (par exemple, courir les soldes en «tennis » et, le soir venu, porter une paire de talons aiguilles). La marche en talons hauts, exclusivement féminine', traverse ainsi toutes les classes sociales et les générations, bien que l'on puisse, dans la pratique, borner l'âge d'entrée vers l'adolescence et de sortie vers le troisième âge.

7 Malgré les améliorations successives - notamment l'invention du cambrion ${ }^{10}$, qui permet de placer les talons à l'extrémité postérieure de la semelle (XIX ${ }^{\mathrm{e}}$ siècle), et le renforcement de la solidité du talon par une âme métallique (milieu du xxe siècle) (Bossan 2004: 76) -, la marche en talons hauts présente toujours une efficacité moindre que la marche à plat. En effet, à partir d'une certaine hauteur, les talons modifient radicalement les appuis du pied et, par là, le dérouler du pas : sept centimètres de talon raccourcissent le pied de cinq centimètres et engendrent une forte pression au centimètre carré (Fombonne 2008 : 317) ${ }^{11}$. Plus les talons sont hauts, moins l'enjambée est étendue. En outre, compte tenu de la courbure de la chaussure, le pied tend à glisser vers l'avant, d'où l'invention de dispositifs - d'une efficacité toute relative - pour compenser ce glissement et réduire l'inconfort des chaussures à talons, dû notamment à l'échauffement des coussins plantaires ${ }^{12}$. Le port de telles chaussures sur des temps longs et/ou de longues distances s'avère fatigant, causant même couramment des lésions orthopédiques et fonctionnelles bien connues (hallux valgus, cors, tendinites...), à tel point que la fédération des syndicats britanniques, jugeant les talons hauts inappropriés dans l'environnement actuel de travail, mit à l'ordre du jour de son congrès de 2009 le projet d'une limitation de la hauteur des talons à deux centimètres et demi pour les femmes qui travaillent debout, et à quatre centimètres pour celles qui passent la journée principalement assises ${ }^{13}$. Certaines femmes usent d'ailleurs 
de stratégies pour pallier les douleurs et l'inconfort des escarpins : choix de la hauteur de talons en fonction de la durée du port (journée, demi-journée, soirée...), de la longueur des trajets et/ou du mode de transport (bus, métro, voiture...), prévision d'une paire de chaussures de rechange...

Porter des talons hauts exige en outre de déjouer les pièges que représentent les surfaces irrégulières (pavés, gravillons...), striées (grilles, escalators, parquets...) ou trop souples comme les pelouses, d'où la fameuse démarche titubante de Rachida Dati, alors Garde des Sceaux, lors d'une garden-party présidentielle ${ }^{14}$. Enfin, au-delà d'une certaine hauteur (dix centimètres environ), conserver son équilibre se révèle ardu; en témoignent les chutes fréquentes des mannequins durant des défilés, obligées parfois d'ôter leurs chaussures ${ }^{15}$, ou celle récente, largement médiatisée, de Mylène Farmer ${ }^{16}$, se soldant par la fracture d'un orteil ${ }^{17}$, ou encore celles des concurrentes des courses en escarpins ${ }^{18}$. Ces problèmes d'équilibre n'ont rien de nouveau : sous Louis XVI, les porteuses de talons hauts étaient obligées de se munir d'une canne pour se stabiliser (Perrot 1984: 75); ils résultent de la réduction de la surface d'appui et de la modification de la position du centre de gravité qu'engendre le port de talons, cette réduction et cette modification s'accentuant avec la hauteur.

La monte en amazone comme la marche avec des talons hauts correspondent au choix incongru de techniques offrant une emprise moins grande sur la nature. Un tel choix contredit la tendance adaptative de l'évolution générale des techniques et constitue ainsi une forme d'« exaptation ${ }^{19}$ technique.

\section{Un échantillon de techniques limitantes}

8 La découverte de la dimension limitante de ces deux techniques me porta à élargir la recherche, dans l'espace et dans le temps, aux autres techniques présentant les mêmes caractéristiques, c'est-à-dire aux techniques qui, dans une même société et pour le même but visé, présentent un caractère limitant, connu des acteurs sociaux, alors même qu'au moins une autre technique plus performante est théoriquement à disposition, mais de fait réservée à certaines catégories de la population.

Une fois constitué, en partie grâce aux Human Relations Area Files ${ }^{20}$, un échantillon aussi représentatif que possible de ces «techniques limitantes », j'ai étudié chacune d'elles : 1) en elle-même, notamment à travers l'analyse des gestes qui sont nécessaires pour la mettre en œuvre, afin d'identifier en quoi elle s'avère limitante ; 2) par rapport aux personnes qui l'utilisent, pour déterminer à quelles différenciations - sexuelle, sociale, ethnique, de classe d'âge... - elle correspond éventuellement ; 3) par rapport à la totalité du système social et culturel considéré, pour tenter d'en dégager une signification plus générale (cf. Mauss 1967 [1947] : 26-27).

10 La principale difficulté rencontrée dans cette entreprise tient à la description des gestes techniques, rarement aussi détaillée qu'il aurait été souhaitable dans les sources explorées. La simple évocation, par exemple, du port de « lourds bracelets de cheville » ne suffit pas pour évaluer les contraintes qu'entraîne ce port; d'autres précisions seraient nécessaires : poids des bracelets, description de la démarche, etc. Avant moi, Paola Tabet, dans son étude sur les outils et les procédés de travail des femmes (1979:22), avait déjà déploré l'absence de description fine des techniques dans les travaux ethnologiques. 
Lorsqu'elle évoque les techniques de chasse du guanaco chez les Ona de Terre de Feu, Paola Tabet oppose l'emploi d'arcs et de flèches par les hommes, à celui de chiens par les femmes, et voit dans le recours à ces animaux un exemple du «moindre équipement féminin » (ibid. : 13). Or, pour conclure à une efficacité et à une complexité moindres de cette technique, il aurait fallu, en toute rigueur, mieux connaitre les caractéristiques du gibier, son mode de vie, le relief du terrain de chasse, la composition et la densité de la végétation, le type des chiens utilisés, leur nombre... En fonction de ces éléments, chasser avec des chiens peut s'avérer plus efficace techniquement et plus complexe que de chasser à l'arc.

Le corpus de techniques présumées "limitantes » ainsi recueilli m'a permis de dresser une typologie dont je ne livre ici, faute de place, que l'armature illustrée d'exemples. Se distinguent deux grandes catégories de «techniques limitantes». La première regroupe des techniques qui, soit par des modifications corporelles intentionnelles ${ }^{21}$, aussi bien réversibles qu'irréversibles, soit par le recours à des artefacts, entravent la mise en jeu du «premier et plus naturel instrument de l'homme » (Mauss 1966 [1936] : 372), le corps. Par exemple, les amputations de doigts (comme chez les Indiens Blackfoot, entre autres) restreignent, en proportion du nombre et de l'importance de ces opérations, les "différents modes d'action de la main dans son rôle préhenseur ": accrocher, pincer, saisir, contenir (Leroi-Gourhan 1943 : 44). La seconde catégorie rassemble des techniques "spécialisées à usages spéciaux " (Mauss 1967 [1947] : 55) qui, à l'instar de la monte en amazone et de la marche en talons hauts normalement répertoriées au titre de techniques de transport, présentent un caractère notoirement limitant par rapport à d'autres techniques de la même catégorie.

\section{Les techniques limitant la mise en jeu du corps}

11 La dimension limitante de ces techniques a une portée générale: elle intervient dans toutes les situations qui exigent le concours des segments corporels concernés : jambes pour se mouvoir, main pour saisir, bras pour lancer...

\section{Les modifications corporelles limitantes irréversibles}

Une des interventions corporelles les plus connues dans ce domaine correspond au bandage des pieds ${ }^{22}$. Cette pratique chinoise consistait à raccourcir le pied en repliant les orteils vers l'intérieur, sauf le pouce, et en redressant le bloc astragalo-calcanéen (Chippaux 1990: 547). Durant près d'un millénaire ${ }^{23}$, la coutume préconisaient que les pieds d'une femme adulte mesurent entre treize et dix-sept centimètres, voire moins de neuf centimètres dans les cas extrêmes ${ }^{24}$. Dans la station debout, cette mutilation obligeait les femmes à une recherche continuelle de leur équilibre. Elle n'autorisait, par ailleurs, que des enjambées de très faible amplitude (Tsai 2008: 65). Marco Polo relate ainsi dans ses mémoires que « les filles marchent toujours si doucement qu'un pied jamais ne se pose devant l'autre de plus d'un demi-doigt » (Bossan 2004 : 164). Réservé, dans un premier temps, aux catégories sociales les plus élevées, le bandage des pieds se diffusa progressivement dans toutes les couches de la société chinoise. Dans les familles aisées, il débutait entre l'âge de cinq et sept ans. Les familles moins aisées le repoussaient de quelques années afin de pouvoir bénéficier du travail et de la mobilité des jeunes filles ( Ibid. : 64). Dans les années 1970, des vieilles dames étaient encore là pour témoigner de la 
pénibilité des pieds bandés à l'usine et de la retraite anticipée que leur avait octroyée pour cette raison le pouvoir républicain (Kristeva 1974 : 93).

L'amputation des doigts fait également partie des techniques limitantes irréversibles. Bien qu'elle diminue les capacités de préhension, elle se rencontre à différentes époques ${ }^{25}$ et dans diverses sociétés - Herero, Barula Kodo, Mafalu (Chippaux 1990: 545-546), Blackfoot (Dempsey $1986: 424)^{26} \ldots$ - et s'en tenait généralement à une phalange, voire à un doigt, l'annulaire ou l'auriculaire. Chez les Sioux, les jeunes hommes sacrifiaient, lors des rites de passage, leur auriculaire et souvent aussi leur annulaire gauche, ce qui ne leur laissait que trois doigts pour tirer à l'arc (Chippaux 1990: 545-546). Les petites filles dugum dani (Nouvelle-Guinée) offraient, elles, leurs doigts en hommage aux défunts; aussi, à partir de l'âge de dix ans environ, presque toutes les femmes avaient déjà perdu quatre à six de leurs doigts. Cependant, les pouces n'étaient jamais supprimés, ni au moins les deux premiers doigts d'une main (Tabet 1998:68-69), de sorte que les femmes pouvaient manipuler, pour le travail des jardins, le léger bâton à fouir féminin. En revanche, elles devaient renoncer aux activités qui nécessitent dix doigts, comme manier des haches, tirer avec des arcs et des flèches ou manier les lourds bâtons à fouir, c'est-àdire aux activités généralement réservées aux hommes (Ibid. : 69).

\section{Les modifications corporelles limitantes réversibles}

On trouve à nouveau dans cette catégorie des techniques qui limitent la locomotion ou la préhension. Ainsi l'engraissement forcé des filles bayankole diminue-t-il de façon significative leur capacité à se mouvoir. Dans cette société d'Afrique de l'Est, une fille qui se préparait au mariage était enfermée pendant une année et gavée de grandes quantités de lait. À la fin de ce confinement, elle ne pouvait plus marcher qu'avec difficulté, tout au plus se dandinait-elle. Son état contrastait avec celui des hommes qui étaient bien développés et même athlétiques (Gracia \& Contreras $2008: 65$ ).

es ongles longs, eux, interfèrent sur l'agilité des mains. Leur pratique s'observe à différentes époques et dans différents lieux. À la fin du XVIII e siècle, Monsieur de Guignes s'étonnait de la longueur des ongles des riches Chinois, des lettrés et des mandarins, c'est-à-dire des " gens comme il faut ». Dans son récit de voyage, il décrit la main gauche d'un médecin dont l'ongle le plus long mesurait « douze pouces et demi $\aleph^{27}$ et les autres "neuf et dix pouces »; il souligne également la gêne dans laquelle vivait cet homme, obligé de tenir sans cesse ses doigts dans des petits étuis en bambou, dont l'usage lui avait aminci la peau (Guignes 1808 : 159). L'auteur fait remarquer, par ailleurs, qu'un ouvrier ne pouvait avoir les ongles longs " puisque le travail continuel [l'aurait] bientôt privé de cet agrément » (Ibid.). À l'heure actuelle, les ongles longs sont d'un usage courant chez les femmes occidentales et c'est souvent davantage le souci de les préserver en bon état que la longueur en elle-même qui contraint les gestes de la main. La pose de faux ongles, occasionnelle ou régulière, connaît aujourd'hui en France un certain engouement, comme en témoigne l'ouverture de nombreuses boutiques spécialisées ${ }^{28}$.

\section{Le port d'artefacts limitant la mise en jeu du corps}

Cette sous-catégorie regroupe les parures et les vêtements qui entravent de façon significative les mouvements du corps. Différentes parties sont concernées: membres, buste, tête... Le port de bracelets, présent dans de nombreuses sociétés, limite ainsi la capacité à se mouvoir. Il correspond à une pratique féminine marquant le plus souvent 
l'état d'épouse et l'appartenance à un statut social élevé ; comme au Rwanda où, à la fin $\mathrm{du} \mathrm{XIX}^{\mathrm{e}}$ siècle, les grandes dames de la cour portaient de la cheville au genou des centaines de larges bracelets qui les obligeaient à une démarche chaloupée, censée imiter celle des vaches (Kagame 1969 : 91). Chez les Dan du Sud (Yacouba), les lourds bracelets de cheville des épouses leur donnaient une "démarche de canard» et les mettaient dans l'impossibilité de courir; ils entraînaient des blessures que les femmes tentaient de prévenir par des chiffons. Bien pensés d'un point de vue technique, ces anneaux qui, une fois refermés, ne pouvaient plus être ôtés, étaient percés de trous afin que l'eau puisse s'écouler lors de la traversée d'un ruisseau (Marie 2003 : 17). Dans la société rgaybãt, des bracelets de cheville, véritables «boulets » de près de cinq kilogrammes, « rivaient » les femmes des notables à leur tente (Caratini 1989 : 222-223).

Dans la France du début du XVII ${ }^{\mathrm{e}}$ siècle, c'est le port du corset qui limitait les mouvements des enfants (filles et garçons) de la société distinguée (Vigarello 2001 : 13). Son usage fut remis en cause par les pédagogues $d u$ XviII $^{\mathrm{e}}$ siècle qui lui reprochaient d'entraver la croissance, de gêner la respiration et, plus généralement, la liberté du corps (Ibid. : 33-36). Il perdura cependant pour les fillettes. Le corset faisait partie également de la garde-robe des femmes adultes depuis le $\mathrm{Xv}^{\mathrm{e}}$ siècle, mais c'est au $\mathrm{xIX}^{\mathrm{e}}$ qu'il connut son apogée (Boucher 1983 : 58). Dans les années 1860, il se vendait à Paris plus d'un million de corsets par an (Borel 1992: 105), en dépit de l'avis défavorable des hygiénistes qui dénonçaient les conséquences délétères que ne manquait pas d'engendrer un port régulier (Ibid. : 109) : le corset était accusé de mutiler le corps et de réduire celles qui le portaient à la "passivité » (Perrot 1984: 173). Les femmes des "classes pauvres» ne le portaient d'ailleurs qu'à l'occasion des fêtes, surtout dans les campagnes; travaillant dur, elles n'avaient guère le loisir de se "crucifier la chair ", sauf pour paraitre "comme il faut " les jours chômés (Veblen 1970 [1899] : 121).

Le port du voile peut aussi présenter une dimension limitante, du moins pour les modèles dont les deux pans exigent d'être tenus sous le menton avec une main ou entre les dents lorsque les deux mains sont occupées, comme font les femmes des régions rurales tunisiennes ou les femmes iraniennes portant le tchâdor traditionnel. Cette nécessité de recourir aux mains ou aux dents pour maintenir le voile en place avait d'ailleurs posé un dilemme lors de la révolution islamique de 1978-1979 en Iran : elle empêchait en effet les manifestantes de lever le poing et de crier les slogans contre le shah. C'est ce qui poussa les membres du Hezbollah (parti de Dieu) à élaborer un nouveau type de voile, le maghna'e , sorte de cagoule cousue sous le menton, qui couvrait les cheveux mais laissait le visage découvert et les mains libres, permettant, de la sorte, de concilier militantisme islamiste et respect du code vestimentaire islamique ${ }^{29}$.

La dimension limitante de ces techniques du corps a une portée générale : elle peut intervenir dans toutes les situations (déplacement, préhension, propulsion lancée...) qui exigent la mise en jeu des segments corporels correspondants (pieds, mains, bras...). Il est difficile de mettre en évidence pourquoi une société, à un moment donné, par un « consensus secret » (Borel 1992: 17), en vient à se focaliser sur telle ou telle partie du corps humain. Le point de départ de semblables pratiques reste souvent obscur. Ainsi, l'origine des pieds bandés a donné lieu à différentes versions : imitation du pied-bot d'une impératrice fameuse, influence d'une courtisane adulée, goût d'un empereur pour les pieds effilés " comme des croissants de lune »... (Bossan 2004: 164). Il y a tout lieu de penser que cette pratique a consisté, dans ses débuts, en un simple bandage qui, à l'instar 
d'autres mutilations volontaires, est allé au fil du temps dans le "sens d'une exagération », favorisée par des " conditions culturelles » spécifiques (Chipaux 1990 : 589) et/ou un « milieu favorable » (Leroi-Gourhan $1945:$ :373). On sait par ailleurs que les effets conjugués de l'imitation (Tarde 1993 [1890]) et de la compétition entre individus portent à l'outrance. L'illustration la plus significative de ce processus est fournie par le phénomène des «hyper-types » bien connu des zoo- techniciens : celui-ci résulte de la tendance des éleveurs et des juges, dans les concours d'élevage, à favoriser exagérément les animaux porteurs des traits les plus emblématiques du standard de leur race - arrièretrain surbaissé des bergers allemands, face écrasée des pékinois et des chats persans... -, traits qui se trouvent de ce fait de plus en plus accentués, jusqu'à entraîner parfois des pathologies propres à certaines races - coxarthrose du berger allemand, conjonctivite et rhinite du chat persan... On peut donc imaginer que, sous l'effet de l'imitation exacerbée jusqu'à l'outrance par les rivalités, les pieds des Chinoises aient été rapetissés, les cous des femmes padaoung allongés (Chippaux 1990 : 535), les tailles amincies par les corsets jusqu'aux limites du supportable, l'innovation technique ayant encore, dans le cas de ces derniers, notamment grâce à l'invention des œillets en métal qui permettaient de serrer davantage (Morris 2005 : 199), contribué à cette radicalisation.

\section{Les techniques "spécialisées à usages spéciaux" limitantes}

19 Comme indiqué plus haut, l'équitation en amazone et la marche en talons hauts figurent ici au titre des modes de transport. Ces deux techniques ayant déjà été décrites de façon détaillée, il n'apparaît pas nécessaire d'y revenir.

L'étude de Paola Tabet sur l'activité des femmes dans les sociétés de chasseurs-cueilleurs met en évidence plusieurs cas où, pour venir à bout des mêmes tâches, hommes et femmes disposent d'outils différents, les outils féminins se révélant à la fois plus rudimentaires et moins spécialisés (1979: 23). Ainsi, chez les Esquimaux de la fin du XIX ${ }^{\mathrm{e}}$ siècle, pour la chasse aux phoques, les hommes étaient équipés de harpons alors que les femmes ne disposaient le plus souvent que d'un petit gourdin en bois. De même, pour les oiseaux, elles n'avaient accès qu'à des arcs munis de flèches à pointe globuleuse qui leur permettaient seulement d'étourdir leurs proies (ibid.). Paola Tabet évoque également la coexistence, dans plusieurs aires culturelles, notamment au Maghreb, d'une poterie masculine au tour et d'une poterie féminine entièrement manuelle (technique dite « au colombin »), alors que le tour a un rendement plus élevé, constant et uniforme (ibid. : 42). Une situation quasi identique s'observe pour le tissage : dans certaines régions, le métier à pédales, réservé aux hommes, est largement supérieur, en termes de rendement, au métier vertical ou horizontal à un rang de lisses des femmes (ibid.: 43). Ou encore, la coexistence de balais à manche long, masculins, et de balais à manche court, féminins, obligeant à balayer courbée ou accroupie, est courante dans plusieurs sociétés, africaines et moyen-orientales notamment ${ }^{30}$.

Bien que toutes ces techniques figurent ici dans la même catégorie typologique, elles ne présentent pas toutes le même profil. Alors que certaines, comme la monte en amazone ou la marche en talons hauts, ont connu des perfectionnements qui ont contribué à leur pérennisation sans toutefois leur permettre d'accéder à la catégorie des "techniques efficaces", d'autres, au contraire, semblent figées dans une immobilité absolue, une "stase technique ", tandis que des techniques homologues, assignées à d'autres tranches 
de la même population, continuent, elles, à évoluer, comme le mettent en évidence les travaux de Paola Tabet (1998 : 74-75).

L'examen de cet échantillon de techniques limitantes montre que celles-ci peuvent s'observer dans tous les types de société. Même si leur nature et les contraintes qu'elles exercent varient, elles ont toutes pour dénominateur commun une inefficacité qui contredit la tendance adaptative linéaire du progrès général des techniques. Même si

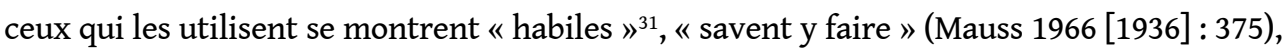
ces techniques n'offrent intrinsèquement qu'une emprise réduite sur la nature. Elles représentent autant de " choix coûteux ", tant pour les sociétés que pour les individus. La portée de tels choix interpelle. Certains chercheurs se sont interrogés sur les conséquences d'une extension de la pratique des pieds bandés à toutes les couches de la société chinoise, en particulier sur la perte de productivité qui en résulterait pour la paysannerie (Damon 2006: 112). Le coût d'une technique limitante a même fait l'objet d'une évaluation précise : la Société française des podologues a estimé que, pour la seule année 2008, les problèmes médicaux liés au port de talons hauts sont à l'origine de quelque deux millions de journées d'absence au travail ${ }^{32}$. Or, force est de constater que, malgré l'inconfort, l'insécurité, les douleurs, voire le handicap qu'elles engendrent, malgré leur inefficacité technique et leur incidence négative sur la productivité, ces techniques ont perduré, parfois sur de très longues périodes, et perdurent encore dans de nombreuses sociétés.

\section{Pourquoi des techniques inefficaces?}

$\mathrm{Au}$ prime abord, les techniques limitantes peuvent apparaître comme autant d'inexplicables bizarreries. Mais on ne saurait se satisfaire de ce premier constat. Ces comportements irrationnels en apparence consistant à se tenir de travers sur un cheval, dans une position instable et périlleuse, alors qu'on pourrait s'installer confortablement à califourchon, à marcher avec des talons hauts dont l'inconfort et le pouvoir pathogène sont connus, quand on dispose par ailleurs de confortables, efficaces et élégantes chaussures plates, à chasser le phoque avec un simple gourdin en bois alors qu'on pourrait le faire bien plus efficacement avec un harpon, dans un contexte où la survie du groupe dépend du succès de cette chasse, etc., doivent bien répondre à une logique. Que signifie l'existence de techniques dont on pourrait dire, pour paraphraser les Shadoks, qu'elles reviennent à faire compliqué quand le plus élémentaire souci d'efficacité ou le simple bon sens commanderaient de faire simple?

\section{Le paradoxe des techniques limitantes}

Comme toutes les techniques, les techniques limitantes s'inscrivent dans un milieu qui ne tient pas au seul milieu technique, mais au milieu social tout entier (Digard 2004 : 202). C'est seulement lorsqu'on s'intéresse à ce dernier qu'apparaissent d'autres dimensions des techniques limitantes. Ainsi, à côté de leurs «fonctions manifestes ", " conséquences objectives comprises et voulues par les participants du système» (Merton 1997 [1957]: 108-135) - ici : maitriser un cheval, se déplacer, capturer et tuer un gibier, tisser, etc. -, ces techniques remplissent aussi des « fonctions latentes ", ni comprises ni voulues (Ibid.), ou "comprises et voulues ", mais toujours en "arrière-plan de la fonction manifeste " (Ferret 2009: 75). Toutes les techniques décrites précédemment remplissent au moins 
une "fonction démarcative» (Bromberger 1979: 116), soit entre les sexes - monte en amazone, talons hauts, pieds bandés... -, soit entre des classes d'âge ou entre des statuts matrimoniaux - bracelets de cheville pour les femmes mariées, engraissement forcé pour les promises, amputation des doigts du jeune chasseur... -, soit entre des classes ou des strates sociales - ongles longs des mandarins, bracelets de cheville des femmes des notables, corsets, pieds bandés avant leur diffusion dans toute la société chinoise... -, soit, enfin, entre des groupes ethniques - femmes padong au cou allongé, Chinoises aux pieds bandés... -, revêtant dans toutes ces situations le caractère de "marqueurs", "traits objectifs [...] retenus et reconnus par les usagers [en tant que] symboles d'identité et d'altérité » (Bromberger 1988 : 90-91). À ce titre, ces marqueurs sont perçus négativement par les étrangers aux cultures considérées, comme en témoignent l'étonnement d'un voyageur oriental devant la manière particulière des Parisiennes de monter à cheval (Al'Amraoui 2002 [1909] : 86) ou la vive réprobation de l'opinion publique occidentale à la vue des Chinoises aux pieds bandés présentes aux expositions internationales d'Osaka (1903) et de Saint-Louis (1904) (Waley-Cohen $2002: 229$ ).

La plupart des techniques décrites manifestent et contribuent avant tout, bien que pas exclusivement, à une "production sociale du genre» (Zaidman 2002 [1977]: 26) et au «travail du genre $»^{33}$. Elles participaient et participent encore, non seulement au maintien de la distinction entre les sexes, mais encore à la hiérarchisation des sexes puisqu'elles pénalisent l'un d'eux d'une emprise moins grande sur la nature. Des techniques comme la monte en amazone ou la marche en talons hauts, pour ne citer qu'elles, sont emblématiques du féminin tel qu'il se définit dans la société occidentale. Elles sont perçues comme constitutives de l'identité féminine et sont revendiquées comme telles par les femmes elles-mêmes (Tourre-Malen 2012b). Ainsi certaines amazones modernes confient que c'est en tant que femmes qu'elles « ressentent l'envie de monter à cheval sous sa forme la plus pure, la plus traditionnelle et la plus féminine »; elles se vivent comme "l'antithèse des cavalières asexuées » qui ont dû renoncer à leur condition de femme pour accéder à ce "sport d'hommes qu'est devenue l'équitation »; la monte en amazone représente pour elles « un moyen de résister à la tendance unisexe qui marque l'équitation actuelle $»^{34}$. On retrouve le même type de propos pour ce qui est des talons hauts. Aussi, le projet des syndicats britanniques évoqué plus haut, tout comme la mise en garde de la Société française de podologie à leur sujet ne reçurent qu'un faible écho ; ils provoquèrent même une réaction de net rejet chez certaines femmes qui voient dans les talons hauts "un outil formidable $\|^{35}$. Les talons hauts représentent en effet un symbole constitutif de l'hyper-féminité largement repris dans les messages publicitaires ; ils sont un révélateur de la féminité, voire son "écrin ", au point qu'afficher de façon aussi ostentatoire cette dernière ne va pas sans gêner certaines femmes qui avouent se sentir davantage "mises à nu " par des talons aiguilles que par un décolleté plongeant. Comme la monte en amazone ou les talons hauts participent de l'identité féminine, les ongles longs étaient constitutifs à la fois de l'identité « professionnelle » et de l'habitus de classe des mandarins.

Que les techniques limitantes remplissent des fonctions latentes ne présente en soi rien de singulier. Jean-Pierre Digard, par exemple, met en évidence, pour l'équitation, des fonctions manifestes - dressage et utilisation des chevaux de selle à des fins identifiées (guerre, chasse, transport, voyage), formation des gentilshommes par l'équitation académique... - et des fonctions latentes qui relèvent de l'ordre du paraître - distinguer le 
noble du roturier, l'officier de l'homme du rang.... L'originalité des techniques limitantes réside dans le fait que leurs fonctions latentes contrarient leurs fonctions manifestes, voire entrent en contradiction avec elles. Les fonctions manifestes de la monte en amazone - contrôler une monture - sont contrariées par ses fonctions latentes : préserver l'«éternel féminin", mais aussi offrir une alternative au chevauchement, symbole de pouvoir des hommes sur le cheval et sur les hommes par le cheval, et du pouvoir des hommes sur les femmes par le biais de la sexualité (Tourre-Malen 2006b : 29). La monte en amazone autorisait les femmes à aller à cheval, tout en évitant un chevauchement féminin longtemps considéré comme contre-nature. On pensait par ailleurs que la position à califourchon - cuisses écartées, sexe au contact de l'animal - était susceptible d'affoler les sens des femmes, et même de provoquer la rupture de l'hymen chez les jeunes filles ${ }^{36}$. La monte en amazone préservant d'un contact trop intime avec la monture rendait la pratique de l'équitation «immaculée » (Ibid.: 27). Elle participait par là au contrôle social du corps des femmes (Ibid.: 32). L'ensemble aboutit au paradoxe d'une technique qui, comme on l'a vu, handicapait ses utilisatrices (Ibid.: chap. I). Les chaussures féminines, elles aussi, remplissent des fonctions manifestes - aide à la marche, protection de la plante et des orteils - et des fonctions latentes - parure du pied, allongement et érotisation de la jambe, exacerbation de la féminité - qui se contrarient à des degrés variables, selon une graduation allant des souliers accessoires de marche aux «souliers-sculptures, moins faits pour être portés que pour exalter ce qu'il y a de plus beau : la cambrure et le cou-de-pied $»^{37}$. C'est également la notion de paradoxe que met en évidence André Leroi-Gourhan dans son étude du kimono japonais. Cas exemplaire, d'après lui, de " désaccord entre la fonction et l'organe ", le kimono lui apparaît comme un «paradoxe» sous l'angle utilitaire (1983 [1946] : 48), n'offrant qu'une protection précaire contre le froid et empêtrant les gestes (ibid. : 46-47). Tout le sens de ce vêtement réside, toujours selon André Leroi-Gourhan, dans sa "fonction figurative ", en tant que symbole des rythmes de la vie, de dignité et d'une "belle image de l'homme » (ibid.: 55). Ce paradoxe entre les fonctions manifestes et les fonctions latentes des techniques limitantes est si caractéristique qu'il convient de définir celles-ci comme des « techniques paradoxales ».

\section{L'efficacité" des techniques paradoxales} latentes. Les ongles longs du mandarin, non seulement le distinguaient des travailleurs manuels de statut subalterne, mais encore le désignaient comme objet de respect: un homme pourvu d'assez « de patience et de raison » pour laisser ses ongles atteindre une telle longueur ne pouvait être soupçonné de se laisser aller à «s'immiscer dans une mauvaise affaire" (Guignes 1808: 160). Le corset, lui, non seulement différenciait les femmes des hommes, mais faisait aussi partie des éléments de la toilette féminine qui attestaient l'exemption du travail productif et la respectabilité aristocratique ou grande bourgeoise (Veblen 1970 [1899]: 118); démonstration de l'incapacité physique des épouses, il témoignait en outre du statut économique et social des maris concernés (Ibid. : 121).

Ce cumul de différentes fonctions latentes entraîne plusieurs conséquences. En premier lieu, lorsque l'une au moins des fonctions latentes apparait socialement valorisante et/ou consiste à marquer des fractures constitutives de la société considérée, le refus ou la 
contestation de la technique paradoxale, par ceux ou celles qui s'y trouvent soumis, seraient assimilés à une déchéance sociale ou à une remise en cause radicale de l'ordre social tout entier.

Ces conséquences expliquent l'un des aspects les plus intrigants des techniques paradoxales, à savoir l'attachement de ceux qui en sont les victimes, et, pour cette raison, les échecs persistants ou répétés des dispositions prises à leur encontre. Ainsi la proscription du bandage des pieds connut-elle de nombreux avatars avant de réussir à s'imposer. En 1664, l'empereur Kangxi décréta l'interdiction absolue de bander les pieds des filles nées après 1662, tout contrevenant à cette loi encourant quatre-vingts coups de bâton et un exil à plus de trois milles (1500 kilomètres); mais ce décret resta sans effet et fut abrogé. En 1694, la même prohibition fut à nouveau promulguée, sans plus d'efficacité (Bossan 2004 : 169). En 1928, le gouvernement de Nankin ordonna encore à toutes les préfectures de veiller à appliquer l'interdiction des pieds bandés, alors qu'ils étaient déjà proscrits depuis plus d'une quinzaine d'années (Damon 2006 : 114). Sans plus de succès, le ministère français de l'Instruction publique en 1902 tenta d'interdire le port du corset que les médecins dénonçaient de longue date (Monneyron 2005 : 28).

Cet attachement aux techniques paradoxales de ceux ou celles qui en sont les premières victimes offre une illustration parmi d'autres de ce que Maurice Godelier a appelé le «consentement des dominés à leur domination» $(1982: 60)^{38}$. Le principe actif du consentement des individus à telle ou telle de ces techniques réside dans le sentiment d'une sorte d'«intérêt bien compris». Pour les Chinoises, le bandage des pieds représentait une préparation indispensable à un beau mariage ; méprisées, les filles aux grands pieds étaient vouées, non seulement au célibat, mais encore aux travaux les plus humbles (Bossan 2004 : 168-169). Plus le bandage des pieds s'ancrait avec le temps dans la culture chinoise comme un canon de l'esthétique féminine et un symbole d'érotisme, plus cette pratique s'imposa aux filles comme socialement bénéfique et son rejet comme pénalisant. En adhérant à cette norme, les familles, sans le savoir, contribuaient à un mécanisme collectif, qui en élargissait les effets (Kaufmann 2005: 78) au point qu'elle devienne constitutive de l'identité féminine chinoise. Le même mécanisme collectif travaille à la pérennisation de la marche avec des talons hauts ${ }^{39}$. Il explique la persistance de telles chaussures dans les garde-robes féminines à une époque qui, paradoxalement, exige des femmes qu'elles se montrent de plus en plus actives, ce dans une société qui tend à l'interchangeabilité des rôles féminins et masculins et qui, par ailleurs, porte une attention croissante au corps, à sa santé, à sa sécurité et à son bien-être. La réputation d'efficacité des talons hauts en termes de séduction est souvent réaffirmée dans les discours féminins : Camilla Morton conseille à ses lectrices d'investir dans une paire de talons aiguilles de dix centimètres pour inciter les invitations à «tomber» (2006:35), tandis qu'une blogueuse confie qu'elle se sent beaucoup plus sûre d'elle lorsqu'elle porte des escarpins car "le regard des hommes est différent, prêt à succomber »; les talons hauts représentent même pour elle un moyen de « prendre le pouvoir » sur les hommes ${ }^{40}$ ; pour d'autres, ils constituent les « vraies sources du girl power $»^{41}$. Revendiqués comme instruments du pouvoir féminin par les unes, les talons hauts sont dénoncés par d'autres comme signes de la domination masculine (Beauvoir 1999 [1949] : I, 205). Il ne s'agit pas ici de trancher entre l'une ou l'autre position, mais de mettre en évidence la diversité des représentations rattachées à cette technique paradoxale. que leur abandon réponde davantage à un changement des mentalités, voire à une 
modification de la structure sociale qu'à des impératifs matériels. Ainsi l'abandon de la monte en amazone, véritable "révolution culturelle", tint à la fois au passage de l'équitation de la sphère militaire à la sphère des sports équestres, à l'évolution de la place des femmes dans la société française et à l'influence étrangère, notamment celle des États-Unis (Tourre-Malen 2006b : 33-49). L'abandon du corset semble lié, selon l'analyse de Thorstein Veblen, au stade d'opulence d'un pays. Dans les pays où la classe de loisir était assez développée et assez riche "pour que toute imputation de travail manuel lui soit calomnie inoffensive et noirceur gratuite ", le corset ne se portait plus. En revanche, il se maintenait dans les pays où l'industrie était encore en train de s'édifier car les classes riches, n'ayant pas encore eu le temps de faire peau neuve, y conservaient les « règles plébéiennes du goût et de l'honorabilité » (Ibid.). L'abandon du bandage des pieds des Chinoises procéderait, lui, du constat, au tournant $\mathrm{du} \mathrm{xx}^{\mathrm{e}}$ siècle, de la faiblesse nationale de la Chine. Il parut alors impérieux aux patriotes chinois de se débarrasser des deux sources de discrédit qui freinaient son ouverture à l'étranger : la dépendance à l'opium chez les hommes et le bandage des pieds chez les femmes (Waley-Cohen $2002: 227$ ). Cette bataille rencontra des résistances : on croisait encore dans les années 1950 des femmes relativement jeunes aux pieds déformés dans certaines provinces de Chine (Chippaux 1990 : 547-551). Tous ces faits montrent bien la force de l'ancrage social et culturel que peuvent prendre certaines techniques paradoxales.

Il reste à envisager une dernière conséquence du cumul de différentes fonctions latentes par une même technique paradoxale. Dans la mesure où la technique paradoxale considérée apparait comme socialement valorisante et/ou constitutive de l'ordre social à un titre ou à un autre, son principe restrictif, loin d'être perçu comme pénible ou dégradant, est souvent, au contraire, recherché, voire accentué jusqu'aux limites du possible, cette dynamique s'ajoutant à celle, évoquée plus haut, résultant des effets conjugués, portant déjà à l'outrance, de l'imitation et de la compétition entre individus. Surtout, le caractère limitant, paradoxal, des techniques en question doit nécessairement être visible, ostentatoire, sauter aux yeux de tout un chacun ; a contrario, son invisibilité ou sa simple discrétion les rendraient inopérantes.

Dans un champ où semblent régner l'efficacité, la rationalité et le progrès linéaire, les techniques paradoxales font figure de monstruosité : au lieu de faciliter les tâches, elles les rendent difficiles ou dangereuses; loin de se montrer efficaces, elles se signalent au contraire par leur pouvoir limitant, leur inefficacité, inefficacité qui apparaît d'autant plus incongrue que ces techniques coexistent, pour les mêmes activités, dans les mêmes sociétés, avec d'autres qui se révèlent, elles, parfaitement efficaces.

Monstrueuses, les techniques paradoxales le sont également sur le plan social puisqu'elles sont réservées et imposées à certaines catégories de personnes, femmes presque toujours, mais parfois aussi lettrés, groupes ethniques particuliers, etc. C'est que, outre leurs fonctions manifestes, matérielles, les techniques paradoxales remplissent aussi une ou plusieurs fonctions latentes, notamment celle de marqueurs identitaires des catégories sociales concernées - fonctions latentes qui viennent souvent contrecarrer les fonctions manifestes, d'où le qualificatif de techniques paradoxales.

Le cumul, par une même technique paradoxale, de plusieurs fonctions latentes ne va pas sans conséquences. En premier lieu, lorsque l'une au moins des fonctions latentes est 
socialement valorisante et/ou marque des fractures constitutives de la société considérée, refuser ou contester ladite technique serait déchoir ou s'exclure. C'est pourquoi les victimes des techniques paradoxales paraissent tellement attachées à leur infériorité technique, attachement qui scelle donc leur aliénation. En second lieu, dans la mesure où la technique paradoxale est socialement valorisante, son principe limitant est recherché et accentué à l'extrême. Son caractère visible, ostentatoire est même une nécessité sociale; a contrario, sa discrétion ou son invisibilité la rendraient inopérante. C'est pourquoi, loin de s'atténuer avec le temps, le caractère limitant des techniques paradoxales va au contraire en s'accentuant, accentuant d'autant, du même coup, l'aliénation de ceux qui en sont les victimes. En résumé, l'efficacité des techniques paradoxales n'est pas technique mais sociale, et cette efficacité sociale n'existe qu'en raison directe de l'inefficacité technique des techniques paradoxales.

Ces techniques paradoxales offrent donc à l'anthropologue un champ de réflexion particulièrement fécond. Elles pointent aussi, une fois de plus, s'il en était encore besoin, l'ineptie de la réduction des techniques aux seuls objets, ainsi que de la distinction classique mais artificielle entre "culture matérielle» et "culture immatérielle». Ces deux travers d'une certaine ethnologie, pourtant maintes fois dénoncés (voir, notamment, Digard 1979), réapparaissent de nos jours, notamment autour des musées et dans le sillage du nouvel engouement pour ces établissements. Il n'empêche: cette réduction et cette distinction représentent de fausses commodités, qui ne feront, aujourd'hui comme hier, qu'amputer les réalités sociales et culturelles d'un de leurs pans constitutifs et qu'atrophier les perspectives holistes qui fondent l'anthropologie.

\section{BIBLIOGRAPHIE}

Al'Amraoui, Idriss, 2002 [1909] Le Paradis des femmes et l'enfer des chevaux. La Tour d'Aigues, Éd. de l'Aube («L'aube poche »75).

Anonyme, 1918 Pour bien faire du sport. S. 1., n. d.

Beauvoir, Simone de, 1999 [1949] Le Deuxième Sexe. Paris, Gallimard. 2 vol.

Borel, France, 1992 Le Vêtement incarné. Les métamorphoses du corps. Paris, Calmann-Lévy.

Bossan, Marie-Josèphe, 2004 L'Art de la chaussure. New York, Parkstone Press (« Temporis »).

Boucher, François, 1983 Histoire du costume en Occident, de l'Antiquité à nos jours. Paris, Flammarion («In-quarto»).

Bouchet, Ghislaine, 1993 Le Cheval à Paris de 1850 à 1914. Genève-Paris, Droz (« Mémoires et documents de l'École des Chartes » 37).

Bozon, Michel, 2001 « Sexualité et genre », in Jacqueline Laufer, Catherine Marry \& Margaret Maruani, eds, Masculin-Féminin. Questions pour les sciences de l'homme. Paris, Presses universitaires de France : 169-186. 
Bromberger, Christian, 1979 « Technologie et analyse sémantique des objets : pour une sémiotechnologie ", L'Homme 19 (1) : 105-140.

Bromberger, Christian, 1988 « Comment peut-on être rashti ?», in Jean-Pierre Digard, ed., Le Fait ethnique en Iran et en Afghanistan. Paris, Éd. du CNRS : 89-108.

Cabaud, Jehanne (Madame Henry [sic]), 1986 L'Équitation en amazone. Paris, Crépin-Leblond.

Caratini, Sophie, 1989 Les Rgaybãt (1610-1934). Paris, L'Harmattan.

Chippaux, Claude, 1990 « Des mutilations, déformations, tatouages rituels et intentionnels chez l'homme ", in Jean Poirier, ed., Histoire des mœurs, 1 : Les coordonnées de l'homme et la culture matérielle. Paris, Gallimard : 483-600.

Closson, Monique, 1992 « La femme et le cheval du XII ${ }^{\mathrm{e}}$ au XVI ${ }^{\mathrm{e}}$ siècles », in Le Cheval dans le monde médiéval. Aix-en-Provence, Publ. du Centre universitaire d'études et de recherches médiévales d'Aix (« Sénéfiance » 32) : 59-90.

Damon, François-Yves, 2006 « Un travestissement impératif : les gardes-rouges », in Guyonne Leduc, ed., Travestissement féminin et liberté(s). Paris, L'Harmattan : 111-122.

Dempsey, Hugh Aylmer, 1986 « The Blackfoot Indians », in R. Bruce Morrison \& C. Roderick Wilson, eds, Native Peoples. The Canadian Experience. Toronto, McClelland \& Stewart : 404-435.

Digard Jean-Pierre, 1979 « La technologie en anthropologie : fin de parcours ou nouveau souffle? », L'Homme 19 (1) : 73-104.

Digard Jean-Pierre, 1994 Le Cheval, force de l'homme. Paris, Gallimard (« Découvertes » 232).

Digard Jean-Pierre, 2004 Une histoire du cheval. Art, techniques, société. Arles, Actes Sud.

Fédération française d'équitation, 2008 Règlement des brevets fédéraux Amazone (document interne).

Ferret, Carole, 2009 Une civilisation du cheval. Les usages de l'équidé, de la steppe à la taïga. Paris, Belin.

Fombonne, Jean-Marc, 2008 Aux pieds des femmes. Érotique du pied et de la chaussure. Paris, Payot \& Rivages.

Godelier, Maurice, 1982 La Production des grands hommes. Pouvoir et domination masculine chez les Baruya de Nouvelle-Guinée. Paris, Fayard («L'Espace du politique »).

Gould, Stephen Jay, 2006 [2002] La Structure de la théorie de l'évolution. Trad. de l'anglais (ÉtatsUnis) par Marcel Blanc. Paris, Gallimard (« NRF essais »).

Gracia, Mabel \& Jesús Contreras, 2008 « Corps gros, corps malades? Une perspective socioculturelle ", Corps $4: 63-69$.

Greer, Germaine, 2002 La Femme entière. Trad. de l'anglais par Edith Ochs. Paris, Plon. Guignes, Chrétien-Louis-Joseph de, 1808 Voyages à Pékin, Manille et l'île de France faits dans l'intervalle des années 1794 à 1801. Paris, Imprimerie impériale. 3 vol.

Kagame, Alexis (abbé), 1969 Introduction aux grands genres lyriques de l'ancien Rwanda. Butare, Éd. universitaires du Rwanda.

Kaufmann, Jean-Claude, 2005 «Le corps dans tous ses états : corps visible, corps sensible, corps secret ", in Christian Bromberger et al., Un corps pour soi. Paris, Presses universitaires de France : 67-88.

Kristeva, Julia, 1974 Des Chinoises. Paris, Éd. des Femmes. 
Laforge, Valérie, 2001 Talons et tentations. Québec, Musée de la civilisation / Montréal, Fides.

Laufer, Jacqueline, Catherine Marry \& Margaret Maruani, eds, 2003 Le Travail du genre. Les sciences sociales du travail à l'épreuve des différences de sexe. Paris, La Découverte (« Recherches »).

Leroi-Gourhan, André, 1943 Évolution et techniques, 1 : L'Homme et la matière. Paris, Albin Michel (« Sciences d'aujourd'hui »).

Leroi-Gourhan, André, 1945 Évolution et techniques, 2 : Milieu et techniques. Paris, Albin Michel (« Sciences d'aujourd'hui »).

Leroi-Gourhan, André, 1983 [1946] « Symbolique du vêtement japonais », in Le Fil du temps. Ethnologie et préhistoire, 1935-1970. Paris, Fayard : 46-55. [Repris de Rythmes du Monde, 1946, 4 : 31-40.]

Marie, Alain, 2003 « La violence faite à l'individu : la communauté au révélateur de la sorcellerie », Politique africaine 91 : 13-32.

Mathieu, Nicole-Claude, 1985 « Quand céder n'est pas consentir : des déterminants matériels et psychiques de la conscience dominée des femmes et de quelques-unes de leurs interprétations en ethnologie ", in L'Arraisonnement des femmes. Essais en anthropologie des sexes. Paris, Éd. de l'École des hautes études en sciences sociales («Cahiers de L'Homme » 24) : 169-245.

Mauss, Marcel, 1966 [1936] « Les techniques du corps », in Sociologie et Anthropologie. Paris, Presses universitaires de France : 365-386.

Mauss, Marcel, 1967 [1947] Manuel d'ethnographie. Paris, Payot.

Merton, Robert King, 1997 [1957] Éléments de théorie et de méthode sociologique. Trad. de l'américain par Henri Mendras. Paris, Armand Colin.

Monneyron, Frédéric, 2005 La Mode et ses enjeux. Paris, Klincksieck (« 50 questions » 23).

Morris, Desmond, 2005 La Femme nue. Trad. de l'anglais par Benjamin Loveluck. Paris, CalmannLévy.

Morton, Camilla, 2006 Comment marcher sur des talons aiguilles. Paris, City Éd.

Nelson, Hilda, 1994 "L'amazone et l'écuyère au XIX siècle ", in De Pégase à Jappeloup, cheval et société. Actes du $5^{e}$ Festival d'histoire de Montbrison. Montbrison, Ville de Montbrison : 101-113.

Perrot, Philippe, 1984 Le Travail des apparences, ou les Transformations du corps féminin, XVIII ${ }^{e}-\mathrm{XIX}^{e}$ siècle. Paris, Le Seuil.

Tabet, Paola, 1979 « Les mains, les outils et les armes », L’Homme 19 (3-4) : 5-62.

Tabet, Paola, 1998 La Construction sociale de l'inégalité des sexes. Des outils et des corps. Paris, L'Harmattan (« Bibliothèque du féminisme »).

Tarde, Gabriel, 1993 [1890] Les Lois de l'imitation. Étude sociologique. Paris, Kimé (« Vues critiques »). Tourre-Malen, Catherine, 2006a « Corps socialisé, corps socialisant », in Michel Biehn, ed., Cruelle coquetterie. Paris, La Martinière : 90-93.

Tourre-Malen, Catherine, 2006b Femmes à cheval. La féminisation des sports et des loisirs équestres : une avancée? Paris, Belin.

Tourre-Malen, Catherine, 2009 « Les contraintes de la féminité », La Grande Oreille. Revue des arts de la parole 37 : 46-50. 
Tourre-Malen, Catherine, 2012a « Pour une ethno-technologie du sport : l'exemple de l'équitation », in Jacques Defrance \& Olivier Holbian, eds, [Titre de l'ouvrage non communiqué]. Paris, L'Harmattan [À paraître].

Tourre-Malen, Catherine, 2012b « L'équitation d'amazone, entre tradition et modernité, entre abandon et réhabilitation? ", in Patrice Franchet d'Espérey, ed., Tradition équestre et pratiques d'aujourd'hui. Saumur, École nationale d'équitation/ Arles, Actes Sud [À paraître].

Tsai, Jacqueline, 2008 La Chine et le luxe. Paris, Odile Jacob.

Veblen, Thorstein, 1970 [1899] Théorie de la classe de loisir. Paris, Gallimard (« Bibliothèque des sciences humaines » 30 ).

Vergnaud, Armand-Denis, 1842 Le Nouveau Manuel complet d'équitation à l'usage des deux sexes. Paris, Manuels Roret.

Vigarello, Georges, 2001 Le Corps redressé. Histoire d'un pouvoir pédagogique. Paris, Armand Colin (« Dynamiques »).

Waley-Cohen, Joanna, 2002 Les Sextants de Pékin. Trad. de l'anglais par Clemence Ma. Montréal, Presses de l'université de Montréal.

Zaidman, Claude, 2002 [1977] « Ensemble et séparés : présentation », in Erving Goffman, ed., L'Arrangement des sexes. Paris, La Dispute : 9-37.

\section{NOTES}

1. À ce propos, je tiens à remercier tout particulièrement Christian Bromberger, JeanPierre Digard et François Sigaut pour leurs encouragements et leurs conseils éclairants.

2. À la différence de ce qui se passe chez les "peuples cavaliers ", dont tous les membres, hommes, femmes et enfants, montent peu ou prou à cheval - à califourchon -, l'équitation est, dans les "sociétés à écuyers", réservée à une élite, généralement masculine, dont elle constitue à la fois l'un des instruments privilégiés de domination et de distinction (Digard $1994:$ 91).

3. Quels que soient l'époque, le type de société, l'animal monté (le cheval bien sûr, mais aussi l'âne, le dromadaire, le renne...) et la manière de monter (emplacement de la selle, diversité des harnachements...), on ne trouve aucun équivalent masculin à la monte en amazone, exception faite des paysans assis en travers le temps de ramener les chevaux de labour à l'écurie après le travail aux champs, en une forme d'« équitation » extrêmement rudimentaire, ce à quoi correspondaient aussi les prémices de la monte en amazone.

4. Un parcours de cross correspond à une série d'obstacles massifs - haies, trous, banquettes... - en forêt ou dans des prés.

5. Sept à neuf brevets fédéraux ou «Galops" jalonnent la progression à cheval dans chacune des disciplines équestres de la Fédération française d'équitation. On retrouve ainsi les Galops de cavalier, les Galops d'attelage, les Galops de voltige, les Galops d'équitation Western, les Galops de pleine nature...

6. En référence au bruit fait pendant la marche.

7. Cf. Restif de la Bretonne, Le Pied de Fanchette ou le Soulier couleur de rose, 1769, cité in Laforge (2001: 61).

8. Hauteur conseillée comme la « hauteur quotidienne » minimum (Morton $2006: 36$ ). 
9. Exception faite des pratiques des drag queens avec leurs chaussures à plate-forme (semelle à épaisseur renforcée, permettant d'augmenter encore la hauteur des talons) et de celles des hommes qui, pour se grandir, portent des talonnettes qui, bien qu'invisibles, peuvent atteindre "jusqu'à 10 centimètres » (http://www.francilux.com, consulté le 8 mai 2008).

10. Le cambrion correspond à une pièce de métal, de bois ou de cuir, qui part du talon et va jusqu'aux métatarses en suivant l'arche de la voûte plantaire. Il a pour fonction de soutenir la voûte et de conserver la cambrure de la chaussure. Jusqu'à son invention, le talon, plus ou moins incurvé, se trouvait vers le milieu de la semelle.

11. Jean-Pierre Fombonne évoque une pression de 2 tonnes $/ \mathrm{cm}^{2}$, mais il semble confondre tonne et kilogramme, ce qui n'est déjà pas négligeable.

12. Cf. : http://www.schollfootcare.com, consulté le 14 octobre 2008.

13. Cf. le site de Libération (www.liberation.fr), consulté le 9 mai 2010.

14. Cf.: http://www.lepost.fr/article/2008/07/17/1225842_rachida-dati-bourree-dansles-jardins-de-l-elysee-impossible-elle-ne-boit-ne-jamais-d-alcool.html\#xtor=ADC-218, consulté le 18 juillet 2008.

15. Cf. le site http://next.liberation.fr, consulté le 15 février 2010.

16. Cf. le site de Libération (www.liberation.fr), consulté le 3 mars 2010.

17. Cf. : http://www.feminactu.com/index.php?q=mylène+farmer, consulté le 7 mai 2010.

18. Cf. : http://www.lemonde.fr/sport/video/2009/11/21/la-course-a-talonscompetition-de-haut-vol_1270473_3242.html, consulté le 21 novembre 2009.

19. Je reprends ici le terme qu'utilise Stephen J. Gould dans sa théorie de l'évolution (Gould 2006 : passim).

20. Je remercie très vivement Sandrine Lecointre, bibliothécaire-documentaliste responsable des HRAF au Laboratoire d'anthropologie sociale du Collège de France, pour l'extrême gentillesse de son accueil et l'efficacité de son aide.

21. Les altérations qui relèveraient de handicaps résultant de maladie ou de traumatisme ne rentrent pas dans le cadre des techniques limitantes telles qu'elles ont été définies.

22. Cette pratique souffre néanmoins de n'avoir fait l'objet que de très peu d'études (Tsai $2008: 63-64)$.

23. On situe l'origine des pieds bandés sous la dynastie Tang (618-907). Leur proscription formelle a été proclamée à l'avènement de la République en 1911 (Chippaux 1990: 547-551).

24. Qualifié alors de «lotus d'or» (Bossan 2004: 169); la taille normale du pied d'une femme se situe entre vingt-quatre et vingt-six centimètres.

25. Pour certains, elle s'observerait dès la préhistoire, mais leurs conclusions sont contestées (Chippaux 1990 : 544).

26. Voir aussi, en ligne, HRAF Document Number : 61 - eHRAF Element Id : nf06-061-00439.

27. Soit presque trente-quatre centimètres.

28. L'époque connaît la vogue du nail art, pratique qui consiste à recouvrir la surface des ongles de dessins fantaisistes; plus de soixante mille sites lui sont consacré, ainsi qu'une encyclopédie (Morris 2005 : 170).

29. Communication personnelle de Jean-Pierre Digard (juillet 2010). 
30. Entretiens avec Christian Bromberger (juillet 2009) et François Sigaut (février 2010).

31. Marcel Mauss précise que le terme " habile » traduit mal le mot latin habilis, bien meilleur selon lui, pour désigner les gens qui ont le sens de l'adaptation de tous leurs mouvements bien coordonnés aux buts qu'ils cherchent à atteindre (Mauss 1966 [1936] : 375).

32. France-Inter, « Journal d'informations » (18h00) du 17 septembre 2009.

33. Pour reprendre le titre de l'ouvrage de Jacqueline Laufer, Catherine Marry \& Margaret Maruani (2003).

34. Cf. : http://amazone2000.free.fr/amazon/pourquo.htm, consulté le 6 octobre 2009.

35. France-Inter, « Journal d'informations » (18h00) du 17 septembre 2009.

36. Ce que l'on pense encore de façon erronée, du moins pour la rupture de l'hymen... (Tourre-Malen 2006b : 170).

37. Texte de présentation de l'exposition Fetish de David Lynch et Christian Louboutin, à la Galerie du Passage en 2007 (http://www.galeriedupassage.com, consulté le 8 août 2008).

38. Malgré la vive critique qu'en a faite Nicole-Claude Mathieu (1985), on s'en tiendra ici à la thèse de Maurice Godelier.

39. Bien que la pression sociale liée au mariage soit sûrement moins forte dans la société française actuelle que dans la Chine d'avant les années 1920.

40. Réaction d'une internaute à l'article «Les pompes à fantasme. Chaussures. Objets de désir et de plaisir, elles rendent certain(e)s accros» (http://www.liberation.fr/ vous/010174400-les-pompes-a-fantasme, consulté le 18 février 2008).

41. L'Express, février 1998 (cité in Greer 2002 : 13).

\section{RÉSUMÉS}

Résumé

L'étude de la monte en amazone et de la marche en chaussures à talons hauts a mis au jour des techniques singulières: les techniques paradoxales. Ces dernières, réservées à certaines catégories de personnes (femmes presque toujours, lettrés, groupes ethniques...), se caractérisent par une inefficacité connue de la société concernée alors que, pour le même but, coexiste une technique à l'efficacité éprouvée. Ces techniques présentent en outre la particularité de posséder des fonctions latentes qui contrarient à divers degrés leurs fonctions manifestes. Monstrueuses à plus d'un titre, les techniques paradoxales, plus encore que par leur inefficacité pratique, se signalent par une efficacité sociale qui s'appuie précisément sur leur caractère limitant: ce caractère est valorisé et accentué aux limites du possible, jusqu'à ce qu'une modification des mentalités et/ou de la structure sociale vienne parfois bouleverser leur reproduction.

Abstract 
The study of sidesaddle-riding in Amazonia and walking in high heels sheds light on a special category : paradoxical techniques. Reserved for certain categories (nearly always women, men of letters, ethnic groups), these techniques are characterized by their ineffectiveness, which the society widely recognizes since other techniques exist with a proven effectiveness for the same purpose. Paradoxical techniques also have latent functions that, to various degrees, hamper the obvious ones. Monstrous in more than one regard, these techniques, beyond their practical ineffectiveness, stand out owing to a social effectiveness based on the limitations inherent in them. This characteristic is valued and stressed up to the limits of what is possible, until a shift in mentalities and/or in the social structure affects the reproduction of these techniques.

\section{INDEX}

Mots-clés : ethno-technologie, techniques paradoxales, monte en amazone, talons hauts, modifications corporelles, distinction de sexe, représentations sociales

Keywords : Ethno-Technology, Paradoxical Techniques, Sidesaddle Riding, High Heels, Body Modifications, Sex Distinction, Social Representations

\section{AUTEUR}

\section{CATHERINE TOURRE-MALEN}

Université Paris-Est Créteil (UPEC), CréteilInstitut d'ethnologie méditerranéenne, européenne et comparative (IDEMEC), Aix-en-Provence 